\title{
FACILE HPLC TECHNIQUE FOR SIMULTANEOUS DETECTION OF ESTROGENTIC HORMONES IN WASTEWATER
}

\author{
${ }^{1 *}$ Muhammad YASIR, ${ }^{1}$ Tomáš ŠOPÍK, ${ }^{1,2}$ Dušan KIMMER, ${ }^{1}$ Vladimír SEDLAŘíK \\ ${ }^{1}$ Centre of Polymer Systems, University Institute, Tomas Bata University in Zlín, Zlín, Czech Republic, EU, \\ yasir@utb.cz \\ ${ }^{2}$ SPUR a.s., Zlin, Czech Republic, EU
}

https://doi.org/10.37904/nanocon.2020.3710

\begin{abstract}
The ever-increasing population has aroused demand for contraceptive chemicals which pollute the water reservoirs through industrial effluents. This has caused a severe threat to marine life and high concern about the availability of fresh drinkable water for people all over the world. Not only this, the unwanted intake of these water containing hormones leads to fertility disorder, rapid femininity, loss in male sperm count, high chances of breast, and ovarian cancer. Therefore, in this study, a one-step high-pressure liquid chromatography (HPLC) technique was devised for simultaneous detection and quantification of various estrogenic hormones from wastewater. Four representative hormones Estrone (E1), $\beta$-Estradiol (E2), Estriol (E3), and 17aEthinylestradiol (EE2) were used. To address the solubility of these hormones, a study was conducted in three solvents systems viz. ethanol, ethanol: water (20:80), and water, respectively. Similarly, calibration curves were made for these three solvent systems with regression coefficients $\geq 0.99$. Interestingly, it was found that water can be used as a solvent for the estrogenic hormones at a detection limit as low as $5 \mu \mathrm{g} / \mathrm{L}$, which makes this study quite significant.
\end{abstract}

Keywords: Wastewater, effluents, contraceptives, estrogenic hormones, liquid chromatography

\section{INTRODUCTION}

Nowadays, in this modern era, manufacturing is increasing in all sectors of the industry due to high demand, pollution is also a huge threat to the people and their environment. Estrogenic hormones such as Estrone (E1), Estradiol (E2), Ethinylestradiol (EE2), Estriol (E3) present in water effluents are a danger to the environment [1]. There have been reported high potencies and significant occurrence of E2, E3, and E1 in flowing waters [2]. Due to their high level of presence, they have been observed and monitored at many wastewater treatment locations [3,4]. EE2 is another synthetic hormone mainly used as an oral contraceptive but also used for the treatment of menstrual problems and prostate cancer. The remains of this hormone are a huge source in the environment because of its partial degradation while treated at sewage plants so its metabolites interact in nature and de-conjugate to become active again [5]. They are classified as endocrine-disrupting chemicals used as medicine for reproduction control and infertility in females, which harms marine life when released in water channels. Studies on marine species have proved fertility disorder, rapid femininity in the fish breed, dropped the weight of testicles in Japanese quails [1]. Also, linkage to humans has proved loss in male sperm count, high chances of breast and ovarian cancer [6,7]. Therefore, it requires proper removal and disposal [5].

In previous works, either one of the hormones was detected and removed whereas, in this paper, the aim is to detect and quantify these estrogenic hormones simultaneously in 3 mediums in a one-step method using the technique of high-pressure liquid chromatography (HPLC) [5]. 


\section{MATERIALS AND METHODS}

\subsection{Materials and reagents}

The estrogenic hormones were estrone (E1), $\beta$-estradiol (E2), estriol (E3), and 17a-ethinylestradiol (EE2) purchased from Sigma Aldrich, Czech Republic. Furthermore, the HPLC solutions: acetonitrile and ethanol of

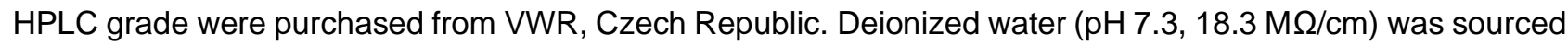
from a laboratory Milli-Q water purification system. Glass microfiber (GMF) filters (Whatman, Czech Republic) with pore size $0.45 \mu \mathrm{m}$ and $25 \mathrm{~mm}$ diameter were used prior to HPLC. Selected values for the properties of the hormones are shown in Table 1 below.

Table 1 Certain properties of hormones [8]

\begin{tabular}{|c|c|c|c|c|}
\hline Estrogenic Hormone & $\begin{array}{c}\text { Molecular } \\
\text { formula }\end{array}$ & Molecular weight $\mathbf{( g / m o l )}$ & Melting point $\left({ }^{\circ} \mathbf{C}\right)$ & Solubility in water (mg/l) \\
\hline Estrone & $\mathrm{C}_{18} \mathrm{H}_{22} \mathrm{O}_{2}$ & 270.37 & $258.0-260.0$ & 12.42 \\
\hline B-Estradiol & $\mathrm{C}_{18} \mathrm{H}_{24} \mathrm{O}_{2}$ & 272.38 & 178.5 & 12.96 \\
\hline $17 \alpha$-Ethinyl-Estradiol & $\mathrm{C}_{20} \mathrm{H}_{24} \mathrm{O}_{2}$ & 296.40 & $182.0-183.0$ & 4.83 \\
\hline Estriol & $\mathrm{C}_{18} \mathrm{H}_{24} \mathrm{O}_{3}$ & 288.38 & 282.0 & 13.25 \\
\hline
\end{tabular}

\subsection{Solution preparation and sampling}

Hormone solution was prepared in three different mediums viz. ethanol, ethanol: water (20:80), and water. For the ethanol, $5 \mathrm{mg} / \mathrm{L}$ stock solution was prepared after stirring solution for $1 \mathrm{~h}$ and it was seen that hormones dissolve immediately. For the ethanol: water system, the concentration of $20 \mathrm{mg} / \mathrm{L}$ in solvent 10:90, $10 \mathrm{mg} / \mathrm{L}$

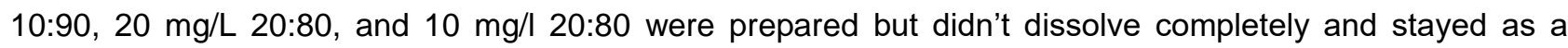
suspension. However, at a higher volume ratio of ethanol: water $20: 80$, solubility was better as can be seen in Figure 1 below.

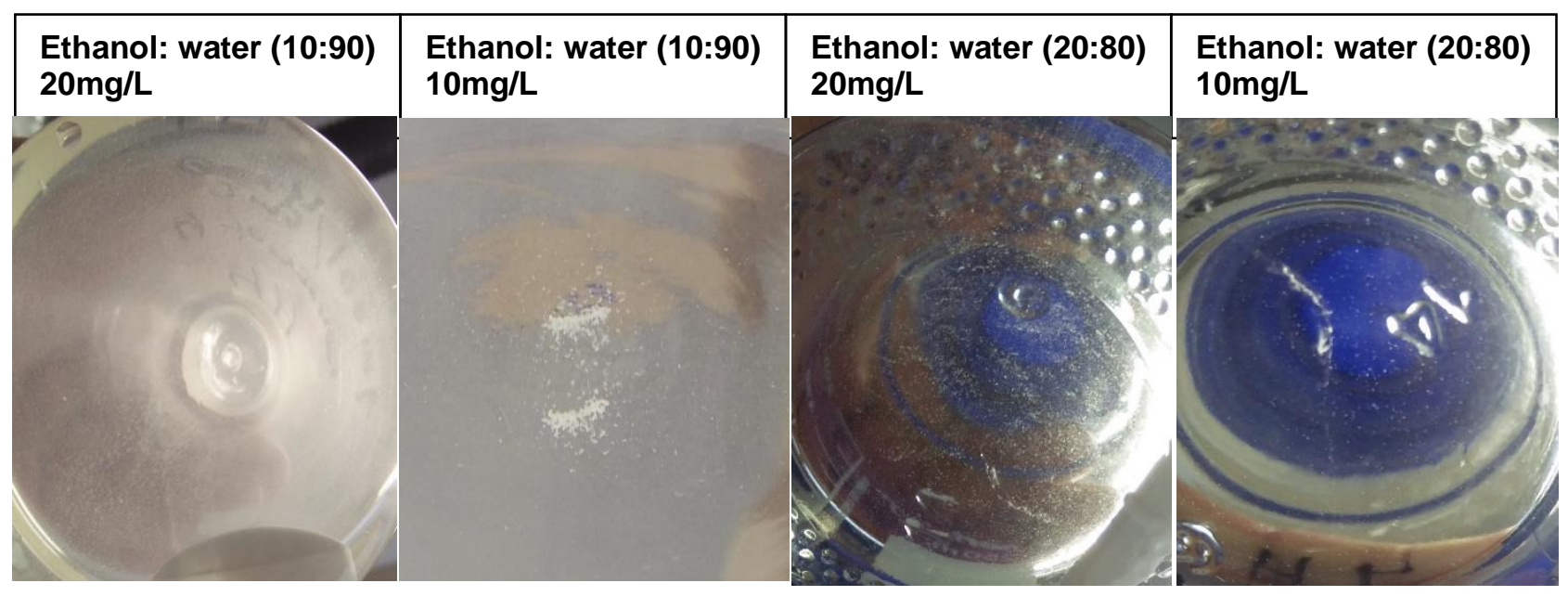

Figure 1 Solubility of all 4 hormones together in a solution at different concentrations

Hence, using ethanol: water $20: 80,5 \mathrm{mg}$ of each hormone was dissolved in a total of $1000 \mathrm{ml}$ by initially dissolving in $200 \mathrm{ml}$ ethanol, followed by $800 \mathrm{ml}$ of water properly stirred at 800 r.p.m for 24 hours to make sure complete homogenous mixing and solubility. It was also observed that if hormones are first dissolved in water followed by ethanol then, hormones are only partially dissolved. The descending order of solubility using HPLC is EE2>E3>E2>E1. In the case of water, solubility was a problem because individual solubility of each hormone is available in literature but considering all 4 hormones together in a single solution was challenging 
especially for distilled and deionized water. Solubility was lower than the values available in the data and it was seen that preparing a solution of $1 \mathrm{mg} / \mathrm{L}$ concentration of each hormone even after stirring for 1 complete day was still having some particles at the bottom of the container which could be referred to as E1 because of its least solubility [7]. It was also checked by individually dissolving each hormone in $1 \mathrm{~L}$ water separately and also when the solution was sonicated for 30 mins, there was an increase in peak for E1 proving that its solubility improved. Therefore, finally, a concentration of $0.2 \mathrm{mg} / \mathrm{L}$ of each hormone was prepared by adding $1 \mathrm{mg}$ of each hormone in $5 \mathrm{~L}$ of water. Samples were collected in $1.5 \mathrm{ml}$ vials after passing through the GMF filter before HPLC and were triplicated to report the mean concentrations with standard deviation for obtaining the calibration.

\subsection{High-Pressure Liquid Chromatography (HPLC) analysis}

HPLC analysis of hormones (E3, E2, EE2, E1) calibration standards and samples were carried out on an HPLC DionexUltiMate 3000 Series (Thermo Fisher Scientific, Germany). The separation was performed on a reversed-phase column Kinetex $2.6 \mu \mathrm{m}$ C18 100 A (150x4.6mm; Phenomenex USA) equipped with a precolumn security guard ULTRA, UHPLC C18 (Phenomenex. the USA) at $30^{\circ} \mathrm{C}$. A mixture of acetonitrile and water was used as mobile phase $(45: 55, \mathrm{v} / \mathrm{v})$ at a flow rate of $0.8 \mathrm{ml} / \mathrm{min}$ with a total isocratic run of $12 \mathrm{~min}$. The sampler chamber was set at $5^{\circ} \mathrm{C}$ and a volume of $20 \mu \mathrm{l}$ was injected into the column. Eluates were detected using wavelengths of $200 \mathrm{~nm}$. Data were recorded and processed in chromeleon 7.2 software (Thermo Fisher Scientific, USA).

\section{RESULTS AND DISCUSSION}

\subsection{Characterization of adsorbent}

To know the surface shape of Hormones, imaging from SEM (FEI, Thermo Fisher Scientific, USA) was carried out. Figure 2 below shows the appearance and surface morphology of each hormone. The figure was captured at $8 \mathrm{kV}$ applied potential and at a magnification of 10000x except for E1 which was 1000x due to the large size of particles.


Figure 2 SEM of a) E3, b) E2, c) EE2 and d) E1 hormones, respectively

\subsection{Detection and quantification}

To identify the peak of hormones, firstly each hormone solution was tested to detect and identify the retention time (r.t), and then a combined solution of hormones was tested. Also, the limit of detection (LOD) and limit of quantification (LOQ) were calculated from $0.01 \mathrm{mg} / \mathrm{L}$ standard as given in the formulas below:

$L O D=\left(\left(\frac{c}{S / N}\right) \times 3\right)$
$L O Q=\left(\left(\frac{c}{S / N}\right) \times 10\right)$ 
Where:

c- concentration (mg/L)

$\mathrm{S} / \mathrm{N}$ - signal-to-noise ratio

Figure 3 represents intensity peaks, calibration equations along with LOD and LOQ of each hormone at their particular retention time in the ascending order of time of E3, E2, EE2, and E1, respectively in a given medium.

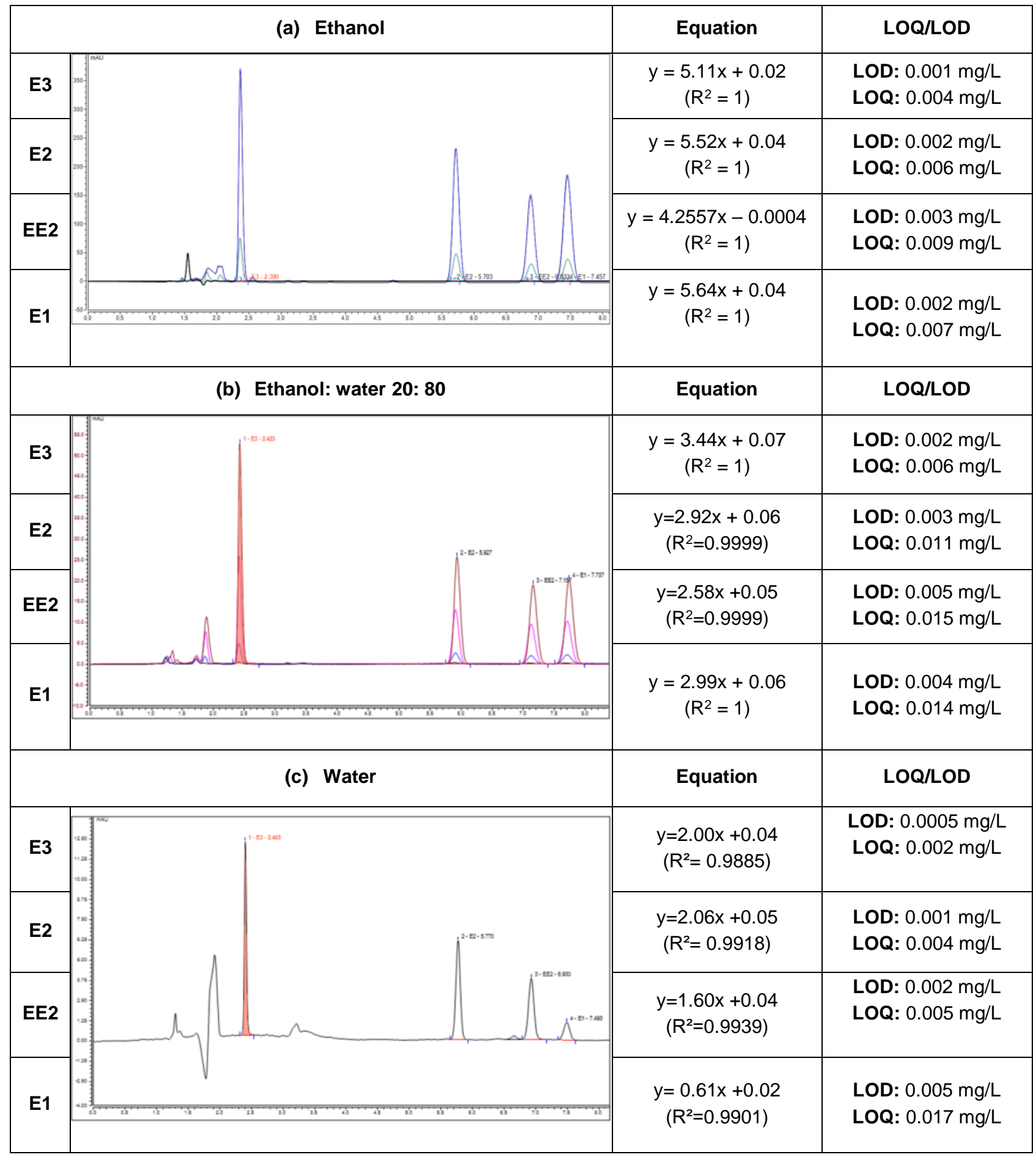

Figure 3 Intensity peak, calibration equation, and LOD and LOQ of each hormone when mixed in a specific solution medium (a) ethanol, (b) ethanol: water 20: 80 , and (c) water 


\section{CONCLUSION}

A method has been developed on HPLC to successfully detect, separately identify and quantify E1, E2, E3, and EE2 hormones together in a single sample with a detection limit of about $5 \mu \mathrm{g} / \mathrm{L}$. It is noteworthy to say that this study has examined only neutral solutions with $\mathrm{pH}$ around 7.3 , which is close to the actual wastewater having $\mathrm{pH} \mathrm{6-9,} \mathrm{and} \mathrm{it} \mathrm{can} \mathrm{later} \mathrm{be} \mathrm{used} \mathrm{for} \mathrm{adsorption} \mathrm{study,} \mathrm{kinetics,} \mathrm{and} \mathrm{also} \mathrm{for} \mathrm{photocatalytic} \mathrm{degradation}$ experiments of these hormones using this method. However, this study has addressed the question of detection and quantification only. Therefore, this study is limited to the presence of hormones in the amounts of micrograms. In general, future research with this approach can also be applied to waste river waters for comparison and to address this issue more deeply and comprehensively.

\section{ACKNOWLEDGEMENTS}

The authors gratefully acknowledge the financial support of the Internal Grant Agency, IGACPS2020002 from the Centre of Polymer System (CPS), Tomas Bata University in Zlin, Czech Republic.

\section{REFERENCES}

[1] NGHIEM, L. D., SCHÄFER, A. I. Adsorption and transport of trace contaminant estrone in NF/RO membranes. Environmental Engineering Science. 2002, vol. 19, no. 6, pp. 441-451.

[2] CARTINELLA, J. L., CATH, T. Y., FLYNN, M. T., MILLER, G. C., HUNTER, K. W., CHILDRESS, A. E. Removal of natural steroid hormones from wastewater using membrane contactor processes. Environmental Science and Technology. 2006, vol. 40, pp. 7381-7386.

[3] JOHNSON, A. C., AERNI, H. R., GERRITSEN, A., GIBERT, M., GIGER, W., HYLLAND, K., JÜRGENS, M., NAKARI, T., PICKERING, A., SUTER, M.J.-F., SVENSON, A., WETTSTEIN, F. E. Comparing steroid estrogen, and nonylphenol content across a range of European sewage plants with different treatment and management practices. Water Research. 2005, vol. 39, no. 1, pp. 47-58.

[4] SARMAH, A. K., NORTHCOTT, G. L., LEUSCH, F. D. L., TREMBLAY, L. A. A survey of endocrine-disrupting chemicals (EDCs) in municipal sewage and animal waste effluents in the Waikato region of New Zealand. Science of the Total Environment. 2006, vol. 355, no. 1-3, pp. 135-144.

[5] HAN, J., QIU, W., CAO, Z., HU, J., GAO, W. Adsorption of ethinylestradiol (EE2) on polyamide 612: Molecular modeling and effects of water chemistry. Water Research. 2013, vol. 47, no. 7, pp. 2273-2284.

[6] SOLOMON, G. M., SCHETTLER, T. Environment and health: 6. Endocrine disruption and potential human health implications. CMAJ. 2000, vol. 163, no. 11, pp. 1471-1476.

[7] HAN, J., QIU, W., GAO, W. Adsorption of estrone in microfiltration membrane filters. Chemical Engineering Journal. 2010, vol. 165, no. 3, pp. 819-826.

[8] TABAK, H. H., BLOOMHUFF, R. M., BUNCH, R. L. Studies on the persistence and stability of natural urinary and synthetic ovulation-inhibiting hormones in untreated and treated wastewaters in Chapter 43 'Steroid Hormones as Water Pollutants II'. Developments in Industrial Microbiology. 1981, vol. 22, pp. 497-519. 